\title{
Conflictos en torno al patrimonio cultural de Ingeniero White (Bahía Blanca, Argentina)
}

\section{Andrés Pinassi ${ }^{1}$ *}

1 Universidad Nacional del Sur (UNS), Departamento de Geografía y Turismo - CONICET, Bahía Blanca, Argentina

Correo electrónico: andres.pinassi@uns.edu.ar

\section{Resumen}

Desde una perspectiva crítica, el patrimonio cultural es entendido como una construcción social, resultado de procesos o mecanismos mediante los cuales un bien histórico-cultural se sacraliza, considerándolo como aquello representativo de una determinada sociedad y que debe preservarse como tal. Ingeniero White, emplazado en el sudoeste de provincia de Buenos Aires (Argentina), no escapa a estos procesos de activación patrimonial, obteniendo como resultado una serie de tensiones entre los diferentes actores vinculados a estas iniciativas. La presente investigación pretende echar luz en torno a los siguientes interrogantes: ¿quién define el patrimonio cultural de la localidad? ¿Qué patrimonio se construye? ¿Qué componentes quedan fuera de esta construcción? ¿La sociedad reconoce aquello legitimado desde las esferas de poder como patrimonio? ¿Esta definición asegura el uso y disfrute de los bienes patrimoniales por parte de la comunidad?

\section{Conflicts surrounding the cultural heritage of Ingeniero White (Ba- hía Blanca, Argentina)}

\begin{abstract}
From a critical perspective, cultural heritage is understood as a social construction, the result of processes or mechanisms through which a historical-cultural element is sacralized, considering it as representative of a certain society and that must be preserved. Ingeniero White, in the southwest of Buenos Aires (Argentina), does not escape these processes of heritage activation, generating tension among the different actors linked to these initiatives. Research aims to shed light on the following questions: Who defines the cultural heritage of the locality? What heritage is constructed?
\end{abstract}

Recibido

febrero de 2018

Aceptado

junio de 2018

\section{Palabras clave}

Conflictos patrimoniales; Patrimonio cultural; Construcción social; Poder político y económico; Ingeniero White

\section{Key words}

Heritage conflicts; Cultural heritage; Social construction; Political and economic power; Ingeniero White

* Posdoctorado en Ciencias Humanas y Sociales (Universidad de Buenos Aires); Doctor en Geografía (Universidad 
What components are left out of this construction? Does society recognize what is legitimized from the spheres of power as heritage? Does this definition ensure the use and enjoyment of patrimonial assets by the community?

\section{Conflitos ao redor do patrimônio cultural do Ingeniero White (Ba- hía Blanca, Argentina)}

\section{Resumo}

Palavras-chave

Conflitos patrimoniais; Patrimônio cultural; Construção social; Poder político e econômico; Ingeniero White
Desde o ponto de vista crítico, o patrimônio cultural é entendido como uma construção social, resultado de processos ou mecanismos através dos quais um bem histórico-cultural é sacralizado, considerando-o como representativo de uma determinada sociedade e que deve ser preservado como tal. Ingeniero White, localizado no sudoeste da província de Buenos Aires (Argentina), não escapa a esses processos de ativação patrimonial, gerando uma série de tensões entre os diferentes atores vinculados a essas iniciativas. Nesse sentido, esta pesquisa tem como objetivo esclarecer as seguintes questões: Quem define o patrimônio cultural da localidade? Qual patrimônio é construído? Quais componentes são deixados fora dessa construção? A sociedade reconhece a legitimidade das esferas do poder como herança? Esta definição garante o uso e o gozo de bens patrimoniais pela comunidade?

\section{Introducción}

Actualmente, los procesos de definición del patrimonio han cobrado gran protagonismo en diferentes sociedades, producto de distintas causas relacionadas con el crecimiento de la actividad turística a escala mundial; con la generación de mecanismos de definición o afirmación de identidades; con la reivindicación de lo local frente a lo global; entre otros factores sociales, económicos y políticos. En este contexto, se legitiman diferentes componentes - naturales y culturales- vinculados a determinados valores $\mathrm{o}$ atributos, que son definidos por ciertos actores sociales en un momento dado y con un fin específico.

Ingeniero White, fundado como localidad portuaria a finales del siglo XIX, no escapa a estos procesos de reconocimiento patrimonial. A escala local, se identifican bienes y manifestaciones culturales producto de los procesos socioeconómicos que ha atravesado la ciudad. Parte de estos elementos han sido legitimados como patrimonio, lo que condujo a contradicciones y tensiones entre distintos actores y sus intereses asociados en orden a diversos factores: la valorización de determinados bienes en detrimento de otros, la participación de distintas esferas de poder en la escala local y el reconocimiento y uso de los componentes históricos por parte de la comunidad. El puerto, sus actividades complementarias, el carácter autónomo de gestión, la relación con la ciudad circundante y los actores sociales implicados, hacen de Ingeniero White un caso de estudio sumamente interesante de analizar. En este sentido, emergen como interrogantes de la investigación: ¿quién define el patrimonio cultural de la localidad? ¿Qué patrimonio se construye? ¿Qué componentes quedan fuera de esta construcción? ¿La sociedad reconoce aquello legitimado desde las esferas de poder como patrimonio? ¿Esta definición asegura el uso y disfrute de los bienes patrimoniales por parte de la comunidad? 
La estrategia metodológica adoptada parte de un enfoque cuali-cuantitativo. Las entrevistas a informantes clave, como representantes del ámbito público local, miembros del Consorcio del Puerto y empresas del sector privado, articuladas con la aplicación de cuestionarios a los residentes, configuran las técnicas utilizadas para la obtención de datos primarios durante el trabajo de campo.

En primer lugar, se presenta una crítica a la conceptualización instituida del patrimonio cultural; para, en una segunda instancia, analizar los conflictos vinculados a la patrimonialización de los bienes culturales en la localidad de Ingeniero White, no sin antes determinar los actores implicados, los componentes definidos como patrimonio y el rol de los residentes en torno a estas legitimaciones.

\section{Patrimonio cultural: puntos de tensión en relación al concepto instituido}

El patrimonio cultural configura un concepto complejo y polisémico, dadas las diversas disciplinas que lo abordan como objeto de estudio. Constituye, además, un vocablo debatido tanto en la esfera científica como en el ámbito de la gestión (Manero y García, 2016), lo cual conduce a diferentes perspectivas de definición y análisis. Se propone aquí echar luz en relación con estas distintas miradas del patrimonio en general y del cultural en particular, a fin de que se pueda comprender de mejor manera aquello indagado en el estudio de caso.

Una de las líneas de conceptualización del patrimonio -que se encuentra fuertemente institucionalizada - es la arraigada en la base etimológica del término, asociado a la herencia o legado de ciertos bienes que reciben los individuos a nivel personal. En un sentido colectivo, el patrimonio cultural se trasforma en el cúmulo de componentes históricos y culturales, materiales e inmateriales, que una sociedad recibe del pasado y que debe legar a generaciones futuras como señas de su identidad. Este concepto, de amplia difusión en el ámbito académico y de la gestión, ${ }^{1}$ ha sido fuertemente cuestionado. En primer lugar, debido al valor particular, innato, ${ }^{2}$ que se les atribuye a estos bienes y expresiones que forman parte de ese pasado. Hace parecer que el patrimonio nace con determinados atributos propios: históricos, artísticos, paisajísticos y simbólicos, sin tener en cuenta que la sociedad es la que construye dichos valores en torno a las obras o a las manifestaciones. Es así que se establecen criterios de valoración para determinar la relevancia de los bienes culturales y poder decir si un determinado componente es o no es patrimonio. Ejemplo de ello son los inventarios del patrimonio urbano, que realizan una sacralización de ciertas obras que deberían preservarse, fundamentada esta conservación en criterios específicos. Cabe preguntarnos entonces, ¿qué sucede con aquello que queda fuera? ¿No es patrimonio? ¿Quiénes establecen los criterios de patrimonialización? Como se verá en el caso de Ingeniero White, el establecimiento de variables relacionadas con el valor estético, histórico, y aquel asociado al paisaje, son las determinantes, incluso en términos cuantitativos, de la consideración de ciertos elementos muebles e inmuebles como patrimonio.

Otro de los puntos de tensión en torno al concepto institucionalizado se da a partir de la prevalencia del pasado como dimensión temporal en la determinación de un bien como patrimonial. El patrimonio es pensado como una herencia, que viaja en el tiempo, y constituye fuente identitaria de la sociedad. Si bien el pasado se coloca en primer lugar como temporalidad definitoria del patrimonio, el futuro también adquiere relevancia. Este constituye el horizonte temporal al que dicho legado debe arribar, como testigo de la historia comunitaria. Es decir que se produce un sobrevuelo desde el pasado al futuro, sin considerar al presente como temporalidad fundamental en los procesos
1. Bajo esta línea de trabajo se destaca gran parte de la bibliografía existente (Organización de las $\mathrm{Na}$ ciones Unidas para la Educación, la Ciencia y la Cultura [UNESCO], 1972; Consejo Internacional de Monumentos y Sitios [ICOMOS], 1999; Hernández, 2002; Crespí y Planells, 2003; Pardo, 2008; Querol, 2010; Acebo y Schlüter, 2012). Por citar un ejemplo, Querol expresa que: "El Patrimonio Cultural es el conjunto de bienes muebles, inmuebles e inmateriales que hemos heredado del pasado y que hemos decidido que merece la pena proteger como parte de nuestras señas de identidad social e histórica" (Querol, 2010, p. 11).

2. Prats referencia los criterios de patrimonialización o los valores del patrimonio como asociales, es decir, “que están más allá del orden social y de sus leyes" (Prats, 1997, p. 23). 
3. Ballart y Tresserras (2007) lo definen como mensajero cultural.

4. Bajo esta perspectiva se presentan los aportes de Gutiérrez (2014, 2017) y Pinassi $(2016,2017)$.

5. Estos aspectos han sido abordados en: Troncoso y Almirón (2005); Troncoso (2008, 2012) y Smith (2011).

6. Harvey (2005) referencia a estos procesos como "poderes monopolistas".

7. Santana (2002) establece que la autenticidad buscada por los visitantes no tiene que tener exacta congruencia con aquella pensada y gestionada en el territorio, sino que entran en juego la experiencia y percepción que tiene el individuo sobre la realidad patrimonial que vivencia.

8. En esta línea, Prats (2005) referencia a estos bienes o lugares como patrimonios incómodos; Smith (2011) habla de patrimonios disonantes; $y$ Ashworth y Hartmann (2005, citado en Norrild, 2005) destacan a los patrimonios del horror. de patrimonialización. Es desde el tiempo presente, con una determinada ideología y contexto imperante, que se define y constituye el patrimonio.

La inmutabilidad de los componentes patrimoniales se presenta como otra arista de cuestionamiento. Si bien el patrimonio se configura como un viajero cultural en el tiempo, ${ }^{3}$ no se alude que a lo largo de su existencia puede sufrir trasformaciones, tanto en su fisonomía, como en su funcionalidad y valoración social. Es decir que los valores de los bienes culturales, ya sean de carácter formal, de uso o simbólico-significativo (Ballart, 1997), adquieren cierto dinamismo y fluctúan según las demandas y requerimientos de la sociedad. ¿Qué sucede entonces con esas mutaciones que se van produciendo a lo largo del tiempo en las obras históricas o en las expresiones culturales? ¿También forman parte del valor patrimonial? Ante estos cambios, ¿se desvirtúan los atributos por los cuales un bien fue definido como patrimonio?

Otro punto de conflicto se da en relación con el papel secundario que se les otorga a los sujetos (como seres sociales) en las conceptualizaciones etimológicas. Se habla del objeto o expresión, pero no del vínculo o relación que lo une con la sociedad. Bajo esta perspectiva, el patrimonio se configura como tal únicamente a partir de la aprehensión comunitaria del bien cultural. Es decir, el legado histórico adquiere la connotación de patrimonial a partir de la valoración que los individuos realizan de este. ${ }^{4}$

Además de abordar al objeto o manifestación como protagonista de estas conceptualizaciones, también adquiere gran relevancia la alusión al patrimonio como único elemento representativo de la identidad comunitaria. ${ }^{5} \mathrm{Su}$ preservación constituye la acción clave para definir, redefinir o afirmar ciertas identidades. Esto lleva a considerar a veces al patrimonio como filamento unívoco de la identidad, desconociendo que esta es una construcción cultural y que, como tal, puede estructurarse a partir de otras variables que exceden lo meramente patrimonial. Como sucede con la identidad, también se expresa la existencia de un vínculo estrecho con la autenticidad. En este contexto, se desarrollan procesos de gestión de la identidad arraigados en el patrimonio, que buscan resaltar atributos particulares y únicos para constituir así elementos auténticos que solamente se hallan en un territorio específico. La búsqueda de estos atributos singulares de cada espacio ${ }^{6}$ lleva a que, a veces, se desvirtúen las dinámicas de patrimonialización, lo que conduce a impactos indeseados. Esta visión presenta un fuerte énfasis en el espacio portador de los bienes culturales, sin considerar que la autenticidad es un concepto que también depende de la percepción de los sujetos y que es ajeno a los atributos únicos del territorio. ${ }^{7}$

Por otro lado, desde la perspectiva institucionalizada, los componentes patrimoniales pueden distinguirse a partir de dos visiones: una positiva y otra negativa. En el primer caso, las visiones positivas consideran a aquellos bienes que representan valores excepcionales para la comunidad y que deben ser preservados como tales; mientras que en la arista negativa, el patrimonio se asocia a ciertos hechos considerados perjudiciales para la sociedad (atentados, guerras, catástrofes), por lo que la concepción patrimonial es vinculada a un valor ya no excepcional, sino a uno de carácter pernicioso. ${ }^{8}$ Estas distinciones llevan a considerar dos tipologías patrimoniales que parecen ser diferentes, pero que en términos generales representan un mismo proceso de construcción cultural. Estos mecanismos, propios de la versión patrimonial dominante, conducen a una segregación histórica (Smith, 2011), que no tiene en cuenta que, en realidad, todos estos hechos forman parte del mismo devenir espacio-temporal de una determinada sociedad.

Como último punto de tensión, en torno a esta perspectiva instituida, se presenta la contradicción entre el discurso que se promueve por parte de los actores dominantes, vinculado a la idea de un legado que representa a la sociedad y que, por lo tanto, debe 
ser conocido y disfrutado por todos los individuos; y una realidad patrimonial discordante, en la que, en la mayoría de los casos, el acceso y uso de estos componentes por parte de la sociedad resulta nulo o limitado, debido a cuestiones políticas, culturales o de dominio de los bienes. En este sentido, ¿resultan patrimonio aquellos bienes que no son usados o aprovechados por los ciudadanos? Este interrogante funciona como punto de disrupción en el caso de Ingeniero White, debido a que la mayor parte de los componentes definidos como patrimonio se emplazan sobre el sector costero, bajo jurisdicción portuaria, por lo que el libre uso y disfrute del sitio y los componentes patrimonializados que se encuentran en éste queda supeditado a los agentes que ejercen autoridad sobre dicho espacio.

Ante esta lectura dominante que se realiza del legado histórico-cultural, se plantea una perspectiva alternativa, con una fuerza más crítica, que lleva a entender al patrimonio desde otro ángulo. Es así que este se presenta como una construcción sociocultural, elaborada por determinados actores sociales, en una dimensión espacio-temporal dada, con un fin específico. De este enunciado se desprenden ciertos interrogantes, cuya respuesta lleva a clarificar esta mirada alternativa del patrimonio: ¿qué entendemos por construcción sociocultural? ¿Quién la elabora? ¿Cuál es el rol de la comunidad? ¿Qué temporalidad cobra relevancia en estos procesos?

En este enfoque, el patrimonio cultural es considerado como el resultado de procesos sociales, materializados mediante la gestión sobre ciertos componentes históricos (materiales e inmateriales), seleccionados a partir de la determinación de criterios particulares de valoración (históricos, simbólicos, artísticos, paisajísticos), que son establecidos bajo un discurso o ideología y que responden a los intereses del actor promotor de la patrimonialización. Estos mensajes que se transmiten asociados a un bien cultural que es definido como patrimonio responden a un objetivo buscado por sus "creadores"; es decir, determinados agentes sociales que revisten un poder (político, económico, religioso, científico) sobre la sociedad. En estas iniciativas, la comunidad adquiere un rol más pasivo, y se transforma en legitimadora de aquello que ha sido sacralizado como patrimonial. De esta manera, el patrimonio es entendido no como el bien cultural en sí mismo, sino como el proceso por medio del cual se lo define como tal. ${ }^{9}$

Con relación a la temporalidad, a diferencia de la perspectiva etimológica a partir de la cual se construye la definición de patrimonio, en la que el pasado y el futuro se consideran como dimensiones clave, en este abordaje crítico el presente cobra un rol fundamental. Es desde el presente que se rescata ese pasado, el momento en que se decide qué es y qué no es patrimonio. Es desde el presente que se piensan alternativas o posibles usos, para revitalizar los componentes históricos. Es decir que el "hoy" es la temporalidad clave que define el futuro patrimonial de la sociedad a partir de un recorte de su pasado.

Esta mirada, que escapa a los cánones tradicionales, permite abordar el mundo patrimonial de manera diferencial. Ya no basta con entender la realidad histórica y cultural de una localidad, sino que se debe focalizar en el "detrás de escena" de los procesos de activación. No alcanza con analizar un bien o una expresión de la sociedad, sino los mecanismos por los cuales ha llegado a sacralizarse ese componente, que se transforma en referente patrimonial de dicho periodo temporal. En este contexto, el patrimonio deja de ser un elemento neutro producto de la evolución comunitaria, para transformarse en un instrumento discursivo de gran valor político e ideológico a la hora de llevar a cabo distintas iniciativas en las que entre en juego la propia sociedad.

En el caso de Argentina, estos procesos pueden identificarse en ciertos mecanismos de reconocimiento patrimonial llevados a cabo por el poder político hace algunos años. En estos no entraron en juego los valores formales, de uso o paisajísticos, sino que se
9. Autores como Prats (1997, 1998, 2005, 2006, 2012, 2014); Bertoncello (2008, 2010, 2017); Smith (2011); Giop y Flores (2017) se han manifestado en este sentido. 
posicionaron en el centro de la escena los mensajes construidos en relación con los espacios sacralizados. En este sentido se pueden mencionar aquellos sitios vinculados al conflicto de las Islas Malvinas declarados como lugares históricos, la patrimonialización de los pozos de petróleo en Neuquén o la puesta en valor de edificios asociados a los crímenes de lesa humanidad; asimismo, el reconocimiento del automóvil Sedán ("el justicialista") como bien de interés histórico nacional, o la revalorización de las escuelas gestadas durante el período histórico 1945-1955, estructuran ejemplos fieles, en los que el patrimonio, más allá de configurarse como el mero legado de una sociedad, adquiere un rol protagónico y parcial, en el que los discursos se alinean detrás de una ideología, alejándose de la mirada tradicionalista. Por su parte, la relevancia de Ingeniero White como caso de estudio propuesto en este artículo radica en la articulación de las esferas económica y política como promotoras de las iniciativas de legitimación patrimonial, que evidencian un espacio complejo en el que los mecanismos de patrimonialización cobran un rol fundamental como promotores de mensajes e intereses en torno al sector costero y sus agentes vinculados.

\section{Los conflictos en la construcción del patrimonio cultural de Ingeniero White}

La localidad de Ingeniero White -emplazada en el partido de Bahía Blanca, en el sudoeste de la provincia de Buenos Aires (Figura 1) - surge como resultado de la actividad portuaria. Los primeros desarrollos, de carácter precario, se manifestaron a finales de la década de 1850 en la desembocadura del arroyo Napostá. En 1858 se construyó en este lugar el primer muelle rudimentario, que posibilitaba el ingreso de mercancías procedentes de Buenos Aires con destino a Bahía Blanca. Durante el decenio de 1880, con base en las inversiones del Ferrocarril Sud, se llevaron a cabo las primeras instalaciones, y en 1885 se inauguró el primer muelle de hierro (Bróndolo y Zinger, 1978; Viñuales y Zingoni, 1990). El gran auge del puerto, adicionado a los procesos inmigratorios, condujo a que la localidad comenzara a expandirse, y a la urbanización del área aledaña. Este desarrollo, ligado al ferrocarril y al puerto, implicó la creación de diferentes edificios y equipamiento puntual destinados a funciones vinculantes: elevadores, usinas y talleres, entre otros. Dichas obras, articuladas con aquella arquitectura modesta, de

Figura 1. Localización de Ingeniero White

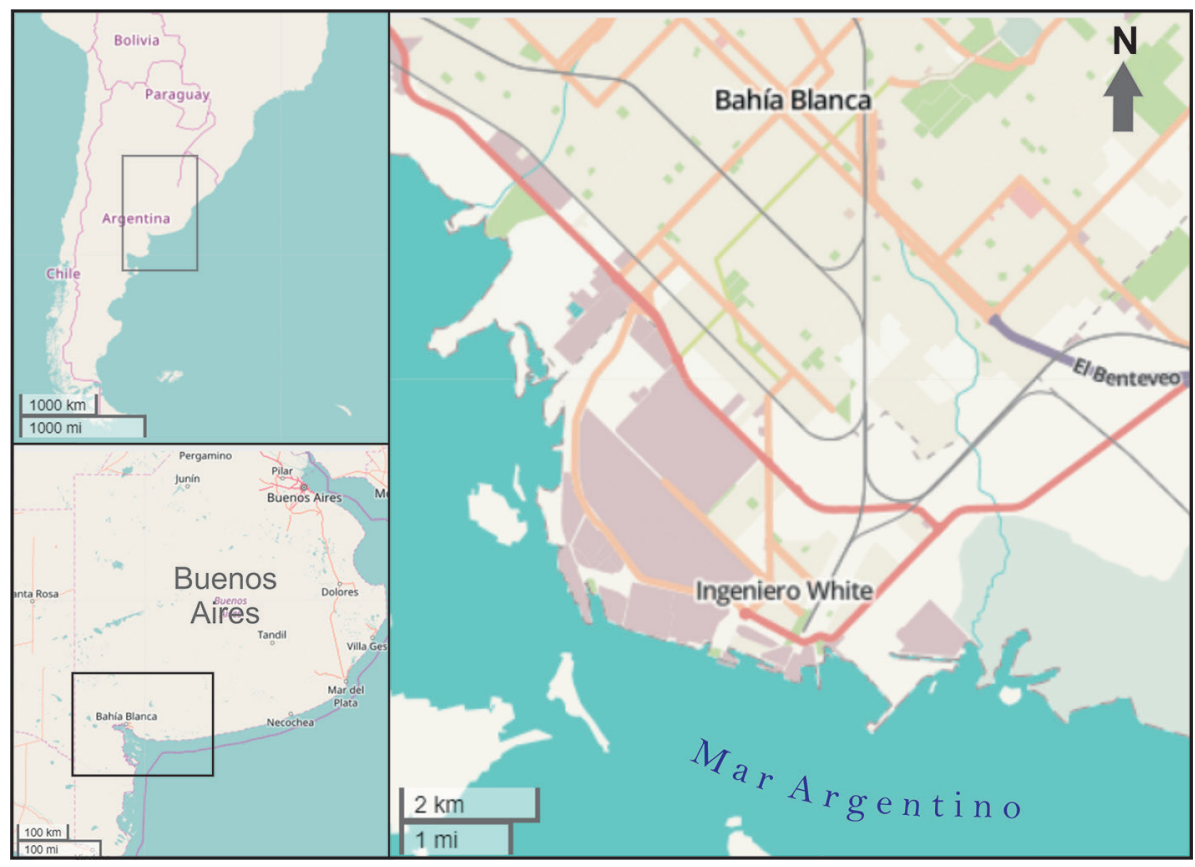


uso residencial (viviendas de chapa y madera y otras posteriores, pertenecientes a los movimientos de vanguardia), adicionadas a las tradiciones y expresiones de los grupos de inmigrantes que se arraigaron allí, constituyen en la actualidad el legado material e inmaterial producto de la configuración socioespacial.

De acuerdo con el objetivo de este trabajo, indagar acerca de los conflictos que se producen en torno al patrimonio cultural local implica determinar aquellos actores clave que incentivaron el conjunto de actividades encaminadas a la selección y sacralización de los bienes culturales que pasaron a formar parte del ideal de cultura del poblado. En Ingeniero White se pueden identificar dos grandes creadores del patrimonio cultural: en primer lugar, el Estado, representado por la Municipalidad de Bahía Blanca, que, a partir de determinados instrumentos de gestión, ha legitimado ciertos bienes en detrimento de otros; y en segundo lugar, algunas de las empresas que estructuran el puerto local y aquellos organismos específicos vinculados a este. Ambos ámbitos de poder comparten espacios comunes de decisión, como es el caso del Consorcio de Gestión del Puerto de Bahía Blanca, que constituye un ente público no estatal, integrado por representantes de los sectores público-privados. En 1992, mediante la Ley № 24.093 se autorizó la transferencia de los puertos de propiedad del Estado nacional a las provincias. Un año después, se creó esta organización, y así, el puerto de Ingeniero White se configuró como el primero de carácter autónomo de la Argentina (Consorcio de Gestión del Puerto de Bahía Blanca, 2017). Este paso de manos públicas a privadas condujo a una segregación espacial entre la propia localidad y el área costera, y despojó a los pobladores de un sector que antiguamente funcionaba como lugar de recreo y esparcimiento. En este contexto, como primera afirmación se puede decir que tanto el poder político como el económico se trasforman en los "constructores" del patrimonio a escala local. En este caso, la población adquiere un rol más pasivo, y es la legitimadora de aquello patrimonializado, a partir del uso social de algunos de estos espacios con determinadas funcionalidades, principalmente de aquellas de carácter recreativo o cultural.

Una de las primeras acciones tendientes a la definición del patrimonio local llevadas a cabo por parte del municipio fue el establecimiento de ciertos sectores susceptibles de valor histórico en la Reformulación del Plan de Desarrollo Urbano de Bahía Blanca, llevado a cabo en 1986 (Municipalidad de Bahía Blanca, 1986). Esta delimitación y selección luego se reafirma con la creación del Inventario del Patrimonio Arquitectónico y Urbano del Partido de Bahía Blanca, elaborado en el año 1992 (Municipalidad de Bahía Blanca, 1992) y ratificado en 1994 por la Ordenanza No 7.959. En el inventario se identifican diferentes áreas, bienes inmuebles y muebles (Tabla 1), que debido a criterios arquitectónicos, históricos y paisajísticos, según lo establecido en el documento, deben ser preservados como tales. 
Tabla 1. Bienes con declaratoria patrimonial a nivel municipal

\begin{tabular}{l}
\multicolumn{1}{c}{ Áreas de valor patrimonial } \\
\hline Conjunto de viviendas de Ingeniero White \\
Puerto Ingeniero White \\
Puerto Galván \\
\hline Inmuebles de valor patrimonial \\
\hline Usina General San Martín \\
Museo del Puerto \\
Vieja Usina del Ferrocarril Sud \\
Subestación ESEBA Galván \\
Subestación ESEBA Ingeniero White \\
Subestación ESEBA Bunge y Born \\
\hline Muebles de valor patrimonial \\
\hline Tableros de electricidad Subestación Galván \\
Tableros de electricidad Subestación Junta Nacional de Granos \\
Equipamiento usina vieja del puerto \\
\hline
\end{tabular}

Fuente: elaboración propia con base en Municipalidad de Bahía Blanca (1992).
10. Como parte del trabajo de campo -llevado a cabo durante el año 2017-, se realizaron diferentes entrevistas a informantes clave de la localidad: representantes de la Delegación Municipal, del Consorcio de Gestión del Puerto de Bahía Blanca, de empresas que intervienen en la comercialización y logística portuaria y a encargados del área de Responsabilidad Social Empresaria de la Unión Industrial. Asimismo, se trabajó con los residentes whitenses. En este último caso, entre los meses de septiembre y noviembre se concretaron un total de 22 entrevistas semiestructuradas a habitantes adultos y adultos mayores. Estas fueron obtenidas a partir de un muestreo dirigido.
Más allá de adjetivar los criterios de valoración de cada sector u obra en particular, interesa analizar, como parte de estos procesos de patrimonialización, qué componentes son seleccionados y con qué historia se corresponden. Se puede decir que la totalidad de ellos pertenecen a áreas y construcciones que tuvieron y tienen alguna función asociada al puerto y al ferrocarril; es decir que, a partir del reconocimiento de estos elementos, se oficializa una historia determinada y se la coloca en primer plano como si fuera ello lo único que tiene valor para preservar como señas de la identidad local. Con este recorte-que, si bien desde el enfoque de la visión patrimonial institucionalizada, puede encontrarse bien fundamentado-, desde la perspectiva que interesa analizar en esta investigación, se están marginando o relegando otras historias (no contadas), vinculadas a los inmigrantes y sus tradiciones, que actualmente son sello del espacio vivido de Ingeniero White.

En relación con las obras declaradas como monumento histórico nacional, se identificaron un menor número de las que cuentan con reconocimiento a escala distrital: la ex Usina General San Martín, legitimada en el año 2002 (Ley 25.580); y los elevadores de granos No 3 y 4 pertenecientes a la ex Junta Nacional de Granos, en el año 2014 (Decreto 2.181). En este contexto, se sigue la misma línea que los bienes declarados a escala local: el legado industrial, de carácter monumental, sigue prevaleciendo sobre otros componentes de carácter modesto y expresiones que configuran el acervo cultural whitense.

Estos patrimonios construidos también son legitimados desde el Estado, a partir de ciertas prácticas sociales que se gestionan en torno a ellos, como por ejemplo, desde el Área de Turismo de la Municipalidad de Bahía Blanca, se ofrece como producto turístico propio de la localidad el "camino de los puertos", constituido por atractivos asociados principalmente a dicha temática. Asimismo, las intervenciones de mejora urbana se relacionan con estos espacios; es el caso de la puesta en valor de la Avenida Guillermo Torres, en el acceso al puerto.

De acuerdo con lo expresado en la entrevista realizada a un representante de la Delegación Municipal de la localidad, ${ }^{10}$ se reafirma que las iniciativas que se vienen desa- 
rrollando desde el municipio se dan en este sentido. Al caracterizar el estado de conservación del patrimonio local, el entrevistado realiza una división en términos de responsabilidades públicas y privadas. Diferencia entre aquel legado en el que el Estado tiene injerencia y el que es de propiedad privada, omitiendo incluso que algunos de estos bienes de dominio particular cuentan con legitimación patrimonial en la esfera normativa, lo que atribuye cierta obligación de custodia al sector público.

Tenemos que dividir en dos partes: una, la que ha tomado a cargo el municipio, que es tanto Ferrowhite, el Museo del Puerto, como la remodelación en el adoquinado histórico en Guillermo Torres, que fue el primer camino que conducía del puerto a Bahía Blanca [...]. Lo que es a cargo del municipio uno lo ve vivo y con futuro. Lo que ha quedado en manos de privados, como las casas de madera, típicas ferroviarias, mezcladas con la cultura pescadora de la localidad, la verdad que dan pena, con lo cual uno [el Estado] puede intervenir hasta cierto punto tratando de concientizar [...], pero no hay voluntad del privado. (Entrevista a miembro de la Delegación Municipal, mayo de 2017)

A pesar de establecer esta diferencia en las responsabilidades y obligaciones, se destaca la importancia que adquiere la comunidad en algunas iniciativas de puesta en valor patrimonial, ya sea de forma particular o a partir de las sociedades de fomento o agrupaciones, como los promotores de ciertas acciones, para posteriormente el Estado posicionarse como apoyo de determinadas iniciativas. Asimismo, destaca que en algunas propuestas -como por ejemplo aquellas que fomentan los paseos turísticos en el espacio histórico costero-, el rol del Consorcio del Puerto es fundamental, debido a que la actividad portuaria es la que ocupa dicha locación -otrora de gran uso socialvinculada al esparcimiento de los residentes.

La Delegación está participando, junto con el intendente, el puerto y las empresas del Polo Petroquímico en la mesa de vinculación llamada: Mesa Ciudad-Puerto. Uno de los ejes de esta es el desarrollo turístico de la localidad, y el Consorcio del Puerto es un actor importantísimo, porque hoy día, el frente marítimo que nosotros conocíamos cuando éramos niños no existe. En este sentido, es el Consorcio el que lleva la delantera en este trabajo. (Entrevista a miembro de la Delegación Municipal, mayo de 2017)

Dicha expresión vuelve a reafirmar no solo la gestión patrimonial pensada en estos espacios clave, de cierta representatividad política, sino también el papel fundamental que adquiere el Consorcio del Puerto como actor relevante en los ámbitos de decisiones.

Respecto de algunas expresiones inmateriales de la cultura -por ejemplo, las festividades arraigadas en elementos propios de la localidad, como la Fiesta del Camarón y el Langostino, las Fiestas Patronales de San Silverio, o el caso de la pesca artesanal-, a diferencia de los sectores históricos construidos, son manifestaciones que quedan relegadas al incentivo y apoyo que les brinde la municipalidad. Si bien se les otorga cierto sustento, en la entrevista se manifiesta que este acompañamiento no se produce en la forma deseable. En el caso de la pesca artesanal, el modelo de gestión autónoma del puerto es el principal elemento que atenta contra dicha práctica socioeconómica identitaria.

En el caso de la Fiesta de San Silverio, además de albergar un valor histórico vinculado a la colectividad de italianos provenientes de la isla de Ponza, en el año 1994 el santo fue legitimado como patrono del puerto y de los pescadores de Bahía Blanca (Puliafito, 2013). Este proceso de reconocimiento extrapola la representación de la figura de San Silverio a gran parte de los pobladores locales, más allá de los residentes con raíces inmigratorias. La nueva forma de administración portuaria -iniciada a partir 
de los años noventa- va a traer también trasformaciones en esta práctica religiosa y cultural, lo que da lugar a ciertos conflictos, al igual que en la festividad del Camarón y el Langostino, que son abordados en el siguiente apartado.

A escala local, la temática patrimonial es utilizada como instrumento de acuerdo o negociación en torno a ciertos conflictos que se producen. Ejemplo de ello es el reclamo constante de acceso al mar entre los pobladores de la localidad y de la propia ciudad de Bahía Blanca, quienes a partir de la privatización del puerto y de la instalación de complejos industriales, fueron despojados del uso casi total del sector costero, con lo cual quedan solamente algunos pequeños espacios que permiten contemplar el estuario. Bajo el concepto de "ciudad-puerto", que tiene como objetivo afianzar el vínculo con los pobladores, el Consorcio de Gestión del Puerto ha estimulado la creación de espacios de ocio, como el Paseo del Puerto y el Balcón al Mar, en torno a lugares históricos, y así otorgó la posibilidad de uso (parcial) a los ciudadanos, de un espacio de dominio privado. No queda lugar a duda de que la reutilización del patrimonio, como expresa Choay (2007), permite en los casos analizados integrar estos componentes a la vida de las personas, y que esto contribuye a la difusión de los valores históricos del área. Pero por otro lado, lo que tampoco puede negarse es que estas iniciativas forman parte de la responsabilidad social de las empresas, que de manera implícita (o intencional), buscan lograr un cambio en la imagen vinculada a los actores privados que despojaron a los pobladores de un uso libre de la costa.

En la entrevista realizada a uno de los miembros del Consorcio, al indagar acerca de cómo definiría a Ingeniero White, desarrolla una adjetivación dicotómica entre progreso y retroceso, y atribuye determinadas problemáticas que se enfrentan en la localidad como parte de las lógicas globales. Esta caracterización de un White contradictorio va a plasmarse luego, cuando el entrevistado se refiera de manera específica al estado de conservación del patrimonio construido.

White es una localidad típica de una zona portuaria que sufrió y sufre las consecuencias de la trasformación económica del mundo y la trasformación de los sistemas laborales y de todo lo que es la implementación de las nuevas tecnologías, entonces no evoluciona, sino que lentamente involuciona [...]. Es un contrasentido, un sector industrial portuario que es muy rico y mueve mucha riqueza y una localidad que no [...]. Entonces, se trabaja para que algo de esa pujanza económica que está alrededor se vea reflejada en la localidad, pero a su vez, la gente tiene que tomar conciencia que hay que construir un nuevo perfil de White, porque tuvo épocas de esplendor, épocas muy buenas, pero esa época ya no va a volver, entonces hay que construir la modernidad de White. (Entrevista a miembro del Consorcio de Gestión del Puerto de Bahía Blanca, mayo de 2017)

Esta paradoja que expresa el entrevistado -acerca de la opulencia de un sector económico próspero que se inserta en un territorio con diferentes problemáticas: sociales, ambientales y culturales- configura un escenario complejo en el que la preservación de los componentes históricos no constituye una prioridad. Asimismo, la construcción de la modernidad que comenta el integrante del Consorcio puede constituir una amenaza contra este legado.

Cuando referencia de forma específica el estado de conservación del patrimonio histórico, el entrevistado limita su definición a aquellos componentes que cuentan con una declaratoria en la esfera normativa. Por otro lado, reafirma la construcción patrimonial realizada en torno a las obras ferroportuarias, distinguiendo entre las que ameritan un mayor cuidado y preservación y las que no; es decir, aquello que tiene cierto valor, representatividad y mayor funcionalidad de acuerdo con los usos contemporáneos. 

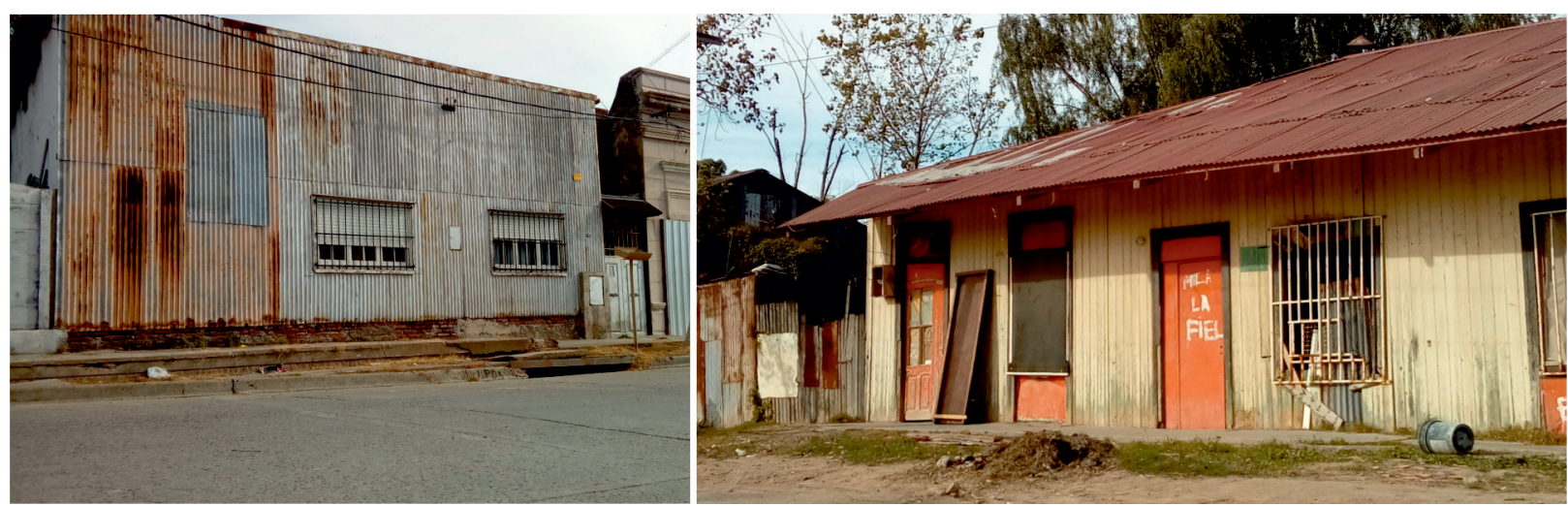

Los edificios que son patrimonio urbanístico tienen una conservación obligatoria, de hecho, dentro del puerto tenemos varios, te diría que la mayoría están dentro del puerto. En Galván tenemos el molino harinero, tenemos un elevador de Oleaginosa Moreno que es patrimonio histórico, por eso no se pueden demoler, sino que se adaptó para poder seguir usándolo, con los inconvenientes de utilizar una estructura vieja. Las casas, estéticamente pueden parecer muy lindas, pero hay que vivir en una casa de chapa y madera (Figura 2). Quedan pocas, van quedando cada vez menos. Entonces, si vos me decís el Museo del Puerto, sí es un edificio histórico, es de chapa y madera y se lo debe preservar como está, pero porque no vive gente. Si me decís casas particulares, te digo que tienden a desaparecer y tienen que desaparecer. La gente no puede vivir como hace cien años. Estamos hablando de construir la modernidad. Esto es lo difícil, el equilibrio entre la nostalgia y lo moderno. Entonces, los edificios públicos que haya que conservar los conservaremos, y las casas de chapa y madera si querés conservar el frente también [...]. Se preserva el aspecto, la imagen, pero no se vive en una casa que no es un módulo habitacional. [...] Es caro mantener la historia y mucho más hacerla convivir con la modernidad. (Entrevista a miembro del Consorcio de Gestión del Puerto de Bahía Blanca, mayo de 2017)

Estas expresiones se traducen en una arena patrimonial de conflicto a escala local, una lucha entre las obras que son consideradas por las esferas de poder como patrimoniales y las que no. Un espacio de tensión entre historia y modernidad, sin considerar que ambas aristas pueden conciliarse. Cabe destacar que, detrás de estas disputas, se encuentran los intereses y la ideología de cada actor, lo que se materializará en la salvaguarda o en la pérdida de determinados bienes.

Por otro lado, las iniciativas que se están comenzando a desarrollar en la actualidad destinan la inversión de recursos en el mismo sector portuario, como expresó el representante. En este contexto, se lleva a cabo un proyecto para darle mayor dinamismo al área costera, comprendida entre el Club Náutico y la ex Usina General San Martín, como parte de un plan integral para el desarrollo de Ingeniero White. ${ }^{11}$

A partir de las consultas llevadas a cabo a algunas de las empresas instaladas en la localidad y a miembros de la Unión Industrial de Bahía Blanca, ${ }^{12}$ se puede afirmar que el ámbito de la preservación de la historia y la cultura les es indiferente; incluso, algunos de estos agentes privados tienen en su propiedad obras históricas abandonadas que cuentan con legitimación en la esfera normativa, como es el caso de la antigua usina del Ferrocarril Sud, sin manifestar ninguna propuesta de refuncionalización. Sin embargo, otras empresas han realizado intervenciones, como en el elevador de la Oleaginosa Moreno, que bregan por mantener en funcionamiento ciertas estructuras históricas, luego de adecuarlas a las exigencias contemporáneas.
Figura 2. Viviendas de chapa y madera correspondientes al periodo de estructuración y consolidación portuaria en la localidad

Fuente: Pinassi (2017).
11. En este sentido, recientemente se han difundido las propuestas de renovación urbana que se llevarán a cabo por parte del Consorcio de Gestión del Puerto de Bahía Blanca en el ingreso al sector denominado Paseo del Puerto, que darán continuidad a los trabajos realizados por el municipio en la Avenida Guillermo Torres.

Asimismo, se ha planificado una intervención en el antiguo muelle de los elevadores, en proximidades de la ex Usina General San Martín, que tiene como objetivo la consolidación de un paseo costero. Para conocer de manera detallada cada una de las iniciativas, ver: periódico La Nueva (2018a; 2018b).

12. Se realizó una entrevista electrónica a la coordinadora de Relaciones con la Comunidad y Responsabilidad Social Empresaria (RSE) de la empresa Bunge Cono Sur (19/05/2017); y a la encargada de la RSE de la Unión Industrial de Bahía Blanca (22/05/2017). 
En términos generales, puede decirse que los procesos de activación patrimonial conducidos por el poder político y económico- se desarrollaron en torno al sector portuario, e implicaron la sacralización de obras que se vinculan de forma directa con esta actividad. Esto conduce a la marginación de otros componentes que forman parte del acervo cultural local, lo que no solo provoca una segregación en las iniciativas de gestión, sino que también contribuye a una desvalorización social de aquellos bienes excluidos.

\section{La visión de los pobladores: ¿legitimadores de lo legitimado?}

El recorte histórico realizado por los actores dominantes en la construcción del patrimonio local se contrapone a la visión de los propios vecinos, quienes expresan que aquellos componentes que permiten contar la historia local exceden los emplazados en el sector portuario. En este contexto, una vecina encargada de realizar paseos guiados por Ingeniero White y participante de diferentes organizaciones en la localidad expresa en la entrevista realizada:

White es un crisol de todas las colectividades que pasaron por ahí, y esto se sigue manteniendo [...]. Hay una cuadra en la Avenida San Martín que me permite contar cómo nació la localidad y no solo en lo arquitectónico, sino con aquella mirada que tiene que ver con la historia, con la cultura, con la comida. [...] esta avenida es referente, porque tenemos en una de las veredas el primer y único conventillo que queda, por ejemplo, [...] al lado tenés una casa Art Decó, después tenés casas de estilo español e italiano, y enfrente, en esa evolución que tiene la arquitectura de chapa y madera, tenés las casas que eran de los ferroviarios [...]. El patrimonio de White es muy identitario con respecto a las colectividades. (Entrevista a vecina de la localidad, mayo de 2017)

La definición de la residente da cuenta de la diversidad de componentes que son reflejo de la historia whitense; incluso se presenta una dicotomía en lo considerado como patrimonial por la vecina y aquello que definieron los representantes políticos y económicos. El puerto y sus construcciones asociadas escapan a la caracterización que realiza la entrevistada, y dan cuenta que el patrimonio de la localidad se erige principalmente sobre la base de los inmigrantes. Cabe destacar que, si bien estas adjetivaciones disgregan el espacio local entre el poblado propiamente dicho y el puerto, y que esta postura se reafirma hoy día por la tipología de propiedad y gestión autónoma de este último, ambos sectores se encuentran íntimamente relacionados, dado que no se pueden pensar históricamente el uno sin el otro. Incluso, cuando se indaga a la pobladora acerca de cuáles considera que son aquellos referentes materiales e inmateriales del devenir local, vuelve a plantear una división entre el puerto y la propia localidad.

13. Como se mencionara, entre los meses de septiembre y noviembre de 2017 , se realizaron un total de 22 entrevistas semiestructuradas, con el fin de indagar acerca del conocimiento y conflictos asociados al legado histórico-cultural whitense. Del total de entrevistados, el $63 \%$ se correspondieron con el género femenino, mientras que el $37 \%$, con el masculino. Respecto del rango etario, la mayor proporción perteneció al grupo de residentes adultos, con el $59 \%$, y el porcentaje restante $(41 \%)$ a los adultos mayores. Los cuestionarios fueron aplicados en el ámbito de la Sociedad Recreativa "La Siempre personales a particulares.

En cuanto a lo material elegiría en lo que es el puerto, porque nos define el puerto, y nos define más allá de la actividad que tenga, a las lanchas pesqueras junto con el muelle de hierro [...], esto sería lo material en el puerto. Si yo ahora me voy a la parte urbanizada, puede ser el primer cine que hubo en la esquina de Guillermo Torres y Belgrano [...], y en cuanto a lo inmaterial, nos quedan los relatos de familia [...], que nos identifican con ciertos lugares [...], o el saber hacer de la pesca, el saber hacer de las redes, de la pesca según las lunas, según el sol, según las mareas, el viento. (Entrevista a vecina de la localidad, mayo de 2017)

La relevancia del sector portuario como espacio histórico también es evidenciada por el resto de los pobladores entrevistados, ${ }^{13}$ a partir de la valoración social que adquieren algunas de las obras que se emplazan en el área (Figura 3), como por ejemplo la ex Usina General San Martín (Figura 4) o los Museos Ferrowhite y del Puerto. Por otro 
lado, el Teatro de Ingeniero White, surgido de la mano de la Sociedad Italiana "Unione Operai", junto con la centenaria institución "Siempre Verde", se colocan entre las más representativas dentro del ejido urbano. Además de estos componentes históricos, los pobladores resaltaron otros, principalmente obras modestas en las que cobran protagonismo las historias comunitarias; o espacios de socialización, como clubes, cantinas, antiguos cines y comercios donde se desarrollaba la vida social whitense. Esto último se contrapone al discurso patrimonial autorizado, como lo define Smith (2011), y da lugar a nuevas representaciones sociales generadas por la propia comunidad, al crear discursos patrimoniales no autorizados, ${ }^{14}$ o legitimados por los poderes que definen el patrimonio en Ingeniero White.
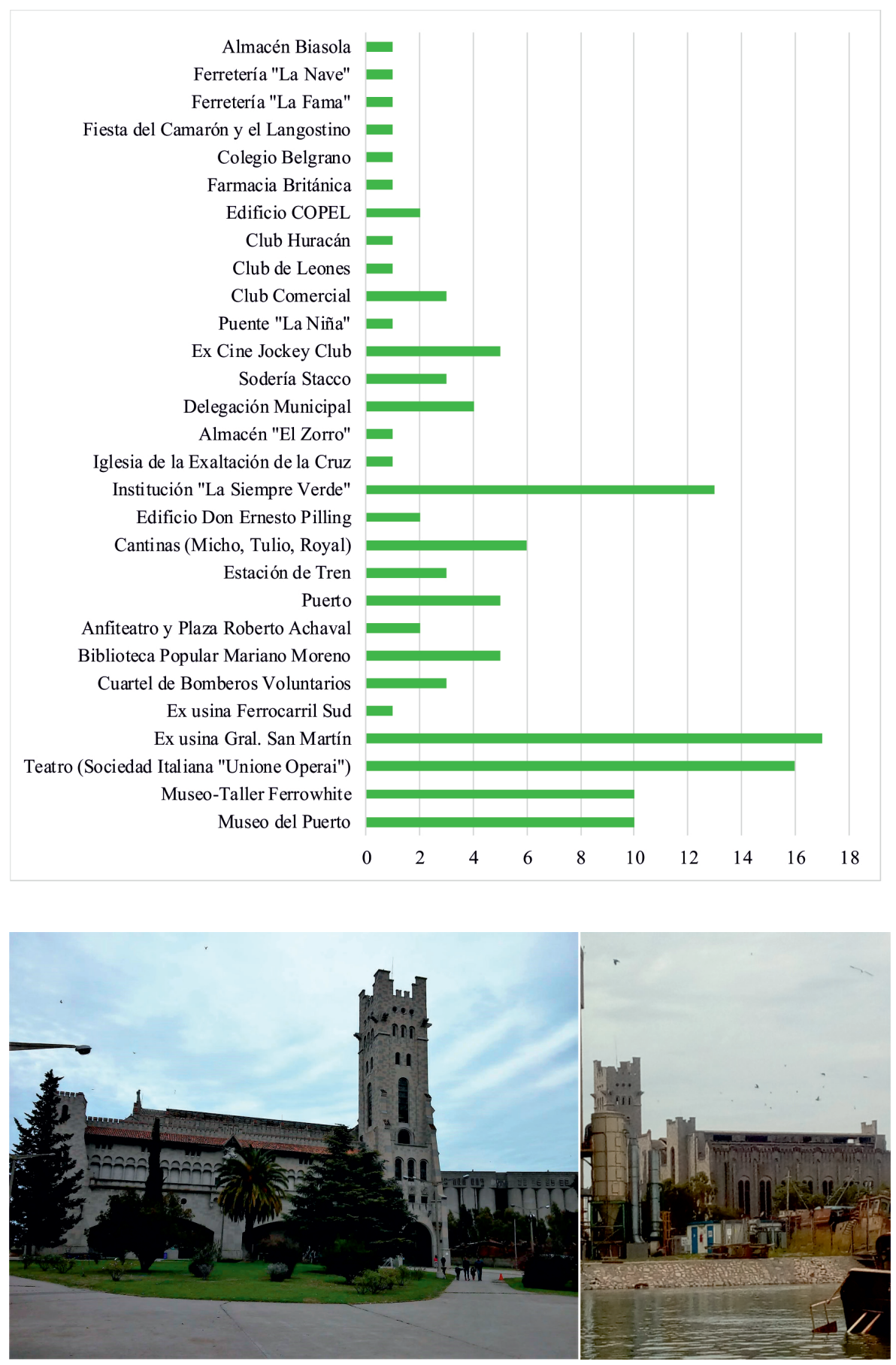

14. "Más allá de este relato oficial que implica el DPA [discurso patrimonial autorizado], existen otros que emergen de la subjetividad e intersubjetividad de los habitantes que, a través de distintas experiencias (temporales y espaciales) producen otros discursos patrimoniales, en este caso 'no autorizados' (DPNA)” (Giop y Flores, 2017, p. 4).
Figura 3. Componentes históricoculturales mencionados por los entrevistados

Fuente: elaboración propia con base en entrevistas realizadas (2017).
Figura 4. Ex Usina General San Martín, parte actual del complejo Museo-Taller Ferrowhite Fuente: Pinassi (2017). 
15. Viviendas destinadas a uso residencial de los trabajadores portuarios durante los primeros años de desarrollo de Ingeniero White.
Si bien se presentan ciertas diferencias entre las consideraciones patrimoniales de los residentes y los representantes del ámbito municipal y del Consorcio del Puerto, también se reconocen puntos en común. Esto se da, por ejemplo, en la percepción de determinados elementos históricos urbanos, como las casas de chapa y madera ${ }^{15}$ que, según los whitenses entrevistados, albergan una acotada valoración social debido a lo que significaba residir en una vivienda de estas características.

La gente a las casas de chapa y madera les tiene hasta cierto odio, o sea de acuerdo a la vivencia [...], la gente grande la asocia con el frío, la asocia con que si los pilotes no eran demasiado altos la casa se inundaba, si la casa se inundaba las maderas se doblaban, y si las maderas se doblaban la casa resultaba inservible, y entonces era pensar en el ingreso de dinero en la familia para poder adquirir otra cosa. [...] Cuando es cuestión de precariedad en lo material, como la chapa y la madera, se tiene una connotación negativa. Entonces, ese patrimonio, que es lo que dio origen a la localidad, se lo desprecia todo el tiempo. (Entrevista a vecina de la localidad, mayo de 2017)

Lo evidenciado en el testimonio de este informante clave se reafirma en las entrevistas realizadas a los pobladores, ya que ninguno de ellos mencionó a estas residencias como componentes representativos de la historia local. Sin embargo, desde el discurso patrimonial autorizado, algunas de ellas guardan un interés histórico relevante, por lo que se las debe preservar. Evidenciado por el inadecuado estado de conservación de estas viviendas y por la carencia de propuestas para su puesta en valor, esta activación patrimonial por parte de los poderes imperantes se desvanece, y queda solamente en la esfera normativa, sin lograr legitimidad ciudadana y fuerza para su institucionalización.

Respecto del estado de conservación del legado histórico de la localidad, la mitad de las respuestas de los entrevistados aludieron a un estado regular de preservación, y señalan la carencia de inversión por parte del Estado o los agentes privados en el mantenimiento de los edificios. Asimismo, el abandono de algunas obras fue una variable reiterada en las respuestas. En este sentido, expresó una de las vecinas: "Los edificios que hoy funcionan están en buen estado, por el cuidado de quienes lo mantienen; no así otros edificios que han sido emblemáticos, pero hoy se encuentran en total estado de abandono y deberían ser considerados patrimonio histórico" (Karina, vecina de la localidad, 2017).

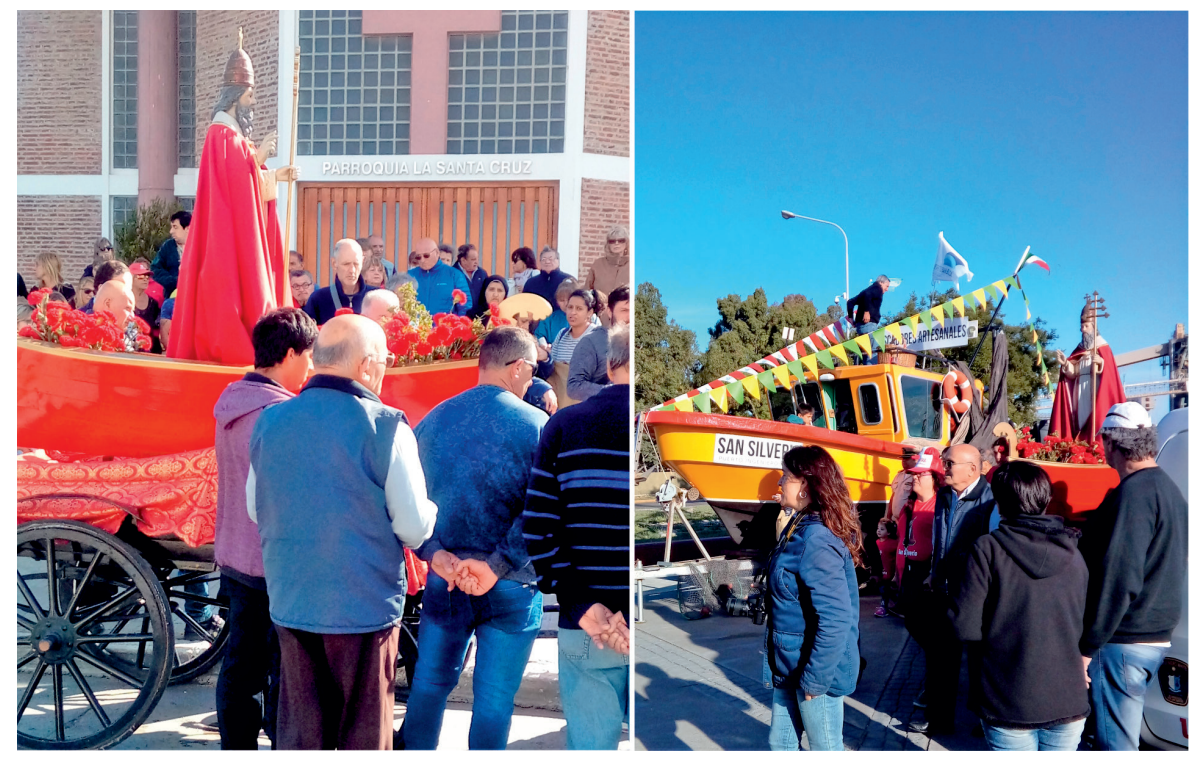

Figura 5. Celebración de San Silverio, en honor al patrono de los pescadores y del puerto Fuente: Pinassi (2017). 
Cabe destacar que un poco más del $30 \%$ de las respuestas aludieron a que estos componentes sí se preservan en adecuadas condiciones. Sin embargo, al indagar nosotros de cuáles de ellos se trata, vemos que son aquellos considerados por el discurso patrimonial autorizado, que en términos generales son los que reciben mayores beneficios para su puesta en valor. Por otro lado, casi el $20 \%$ de las respuestas referenciaron un mal estado de mantenimiento, y destacan el desinterés y el abandono de estos espacios por parte de los responsables públicos.

Al indagar acerca del rol de los actores privados en esta conservación, se manifestó que existen algunas inversiones enclave, como por ejemplo la recuperación de un bar o la instalación de una oficina consular en un edificio histórico, del que solamente se ha respetado su fachada. Sin embargo, se expresa que el vínculo empresas-patrimonio es casi inexistente:

La realidad es que las empresas del puerto nos quieren sacar [...]. La preocupación o el aporte, o las ganas de decir: "esto representa a la comunidad", donde yo también estoy inserto, esas ganas de recuperar algún edificio, no existen. En cuestión de responsabilidad social, ellos pintan las escuelas [...], donan libros a la biblioteca [...], o dan los barbijos; [...] es muy morboso. [...] En cuanto a cuidar el patrimonio no, porque es como la contrapartida de lo que ellos quieren. Si ellos no nos quieren, ¿por qué cuidarían el patrimonio? Porque esto significaría mantener vivo algo que nos identifica y eso haría que uno siga permaneciendo en White; si eso no existe, nosotros nos vamos. Con algo que yo lo veo, es con la celebración de San Silverio [...] (Figura 5). Históricamente cuando la imagen del santo llegaba al puerto, se ponía arriba de una de las lanchas del pescador más antiguo [...]. En algún momento, al Consorcio se le ocurrió [...] que era peligroso que el santo esté arriba de la lancha, entonces, desde hace unos cinco o seis años al santo se lo sube arriba de uno de los buques de Prefectura. [...] Entonces a veces no voy, porque me pone muy mal, porque veo a los pocos pescadores que quedan, y pienso que también nos sacaron hasta la pesca. (Entrevista a vecina de la localidad, mayo de 2017)

Se evidencia, en este sentido, una clara arena de conflicto entre los distintos intereses de cada uno de los actores. Entes privados que, de acuerdo con lo expresado por los residentes, persiguen un fin de lucro, sin importar el espacio vivido de los pobladores de la localidad; y los propios habitantes que, de manera directa e indirecta, dependiendo del caso, bregan por construir un futuro en la ciudad. Estas luchas de poder, de acuerdo con la realidad cotidiana, se evidencian no solo en el ámbito patrimonial, sino en otros planos, como el ambiental: componentes degradados, en inadecuado estado de preservación, que luchan por sobrevivir en un territorio que guarda una fuerte lógica atravesada por preceptos globales, más que locales.

Indagando acerca del rol del Estado de la preservación del patrimonio cultural local, la informante clave entrevistada -al igual que el resto de los residentes- resalta la ausencia del sector público en temas patrimoniales. Los aportes realizados a las festividades locales, como por ejemplo la Fiesta del Camarón y el Langostino, resultan acotados y no fundados en los verdaderos atributos históricos de la manifestación. La encargada de realizar los paseos guiados expresa que, desde hace algún tiempo, en el acontecimiento programado no se desarrolla ninguna actividad relacionada con la pesca y sus materias primas, eje central del evento. Este se ha mercantilizado de tal modo, que se ha desvirtuado el verdadero sentido: reivindicar una práctica tradicional a escala local. Esto mismo puede asociarse a la pérdida de protagonismo de la pesca artesanal, como resultado de la fragmentación del territorio whitense.

En realidad, vos vas a la Fiesta del Camarón y no hay nada alusivo al camarón [...]. Hace mucho tiempo atrás se hacía algo, en algún momento de los días de fiesta, 
alusivo al nombre de la fiesta o alusivo a lo que nos identifica [...], se hacían muestras de cómo se tejía a mano la red, había concursos de quién pelaba más rápido los camarones, cosas que tienen que ver con nosotros, con nuestras raíces [...]. El Estado no aporta en nada [...] estoy hablando de una misma cosa, Estado-empresa en White es lo mismo. (Entrevista a vecina de la localidad, mayo de 2017)

En términos generales, según lo expresado por los entrevistados, se visualiza un fuerte vínculo entre estas festividades y el sentido de pertenencia a Ingeniero White, dado que, tanto la Fiesta Patronal de San Silverio como la Fiesta del Camarón y el Langostino, configuran la representación de una actividad económica de fuerte impronta cultural que caracteriza a la localidad. En este contexto, Oscar expresa: "Mientras subsistan estas festividades culturales y religiosas, White no morirá, es un pueblo de fe de inmigrantes con cultura de trabajo, de sacrificio y con un amor a la localidad que no es negociable" (Oscar, vecino de la localidad, 2017). Sin embargo, el apoyo reducido por parte del Estado y las empresas locales para con estos eventos resurge como una respuesta común entre los habitantes.

Otra de las problemáticas que se plantea en torno a los espacios históricos es la imposibilidad de libre uso que presenta el sector portuario. El modelo de gestión autónoma -desarrollado a partir de los años noventa- constituye la barrera que obstaculiza el acceso a esta área de gran representatividad para los locales. La creación del Paseo del Puerto y el Balcón al Mar por parte del Consorcio da respuesta a esta demanda. En este contexto, se construye un espacio patrimonial que provoca una tensión entre dos dinámicas diferentes: por un lado, la lógica propia de un espacio económico (puerto), portador del funcionamiento de un sistema que se sustenta sobre preceptos globales; y por otro, aquel espacio configurado sobre la base histórica, que funciona como un nexo entre el pasado y el presente whitense. De esta manera, se quiebra con uno de los preceptos que sustenta la noción patrimonial institucionalizada: el uso social del patrimonio por parte de todos los ciudadanos.

\section{A modo de cierre}

Considerar al patrimonio cultural como una construcción social implica posicionarse en el "detrás de escena" de los procesos o mecanismos por los cuales un componente histórico-cultural adquiere la connotación de patrimonial. Mensajes y discursos se alinean con un determinado objetivo ideológico, económico, político, que conducen a la puesta en valor de ciertos componentes en detrimento de otros.

La presente investigación ha contribuido en el análisis de los procesos de construcción del patrimonio cultural en la localidad de Ingeniero White, indagando acerca de las relaciones y conflictos en torno a los actores participantes en la esfera patrimonial. Explorar los vínculos y problemáticas relacionadas con la construcción patrimonial whitense pone en evidencia la existencia de un territorio complejo en el que conviven dinámicas contrapuestas: por un lado, las que bregan por salvaguardar ciertos componentes específicos vinculados a una historia determinada (aquella en torno al puerto y sus espacios adyacentes), y por otro, lo que denominamos como dinámica apática patrimonial por parte de los actores políticos y económicos, que se da en el sector urbanizado. En este contexto, se puede aseverar que los procesos de patrimonialización a escala local responden a los intereses y discursos de estos poderes, y agudizan la segregación espacial producto de la lógica global impuesta por el desarrollo de la actividad portuaria e industrial. En este sentido, se presenta una dicotomía entre lo que según los saberes expertos debe preservarse como señas de la historia local y aquello que no es representativo y que, por lo tanto, no interesa cuidar. El valor formal, configurado por atributos materiales y arquetípicos de los componentes legitimados, 
es utilizado por estos actores sociales como variable relevante a la hora de sacralizar determinadas obras históricas.

Con relación al rol de los residentes en estas activaciones patrimoniales, cabe precisar que en determinados casos, los habitantes funcionan como legitimadores de lo definido como patrimonio; mientras que por otro lado, se visualiza un espacio vivido constituido por componentes que exceden el discurso patrimonial autorizado, lo que da lugar a la valoración de nuevos sitios y de expresiones que escapan a las versiones patrimoniales instituidas. Los comportamientos de consumo cultural y recreativo demostrados por la elevada demanda de ciertos espacios devenidos en patrimonio, como el caso del Paseo del Puerto o de los museos, sustentan esta afirmación; mientras que en una arista diferente, las actitudes reacias a ciertas estrategias llevadas a cabo -principalmente por parte de los actores políticos- da cuenta del carácter activo de los residentes en torno a estos procesos. En este sentido, en las visiones patrimonialistas de los whitenses cobran relevancia los valores de uso y aquellos de carácter simbólico-significativos, atribuidos por los propios pobladores de manera inconsciente a determinados lugares con una carga emotiva y una plusvalía, generada a partir de las vivencias y experiencias en relación con estos espacios.

Se debe destacar que, si bien resultan relevantes las iniciativas desarrolladas en la esfera patrimonial por parte del Consorcio de Gestión del Puerto y por la Municipalidad de Bahía Blanca, la población guarda en su memoria colectiva aquel tiempo en el que el sector costero formaba parte de su espacio de vida, en el que libremente podían transitar y disfrutar de los balnearios del estuario. En este contexto, resignificar este espacio, intentando compatibilizar los discursos de todos los actores implicados, debe constituir el objetivo principal de los procesos de patrimonialización, tratando de quebrar las barreras entre el ejido urbano y el sector portuario, e incorporando nuevos componentes a partir de la visión de los pobladores, quienes constituyen el eslabón clave necesario para batallar los conflictos y tensiones en torno a la definición del patrimonio local.

\section{Financiamiento}

Este artículo es resultado de las investigaciones llevadas a cabo por el autor en el marco de una Beca Interna Posdoctoral del CONICET y bajo el programa de Posdoctorado en Ciencias Humanas y Sociales de la Facultad de Filosofía y Letras de la Universidad de Buenos Aires (Resolución CD № 3.785, Expediente 17.853/17).

\section{Agradecimientos}

El autor agradece la gran colaboración para el desarrollo del trabajo de campo de la Lic. Débora Silenzi. Asimismo, destaca los valiosos aportes realizados por el Prof. Rodolfo Bertoncello en la corrección del documento. 


\section{Q Referencias citadas}

》 Acebo Ibáñez, E. y Schlüter, R. (2012). Diccionario de turismo. Buenos Aires: Claridad.

» Ballart Hernández, J. (1997). El patrimonio histórico y arqueológico, valor y uso. Barcelona: Ariel.

» Ballart Hernández, J. y Tresserras, J. (2007). Gestión del patrimonio cultural. Barcelona: Ariel.

" Bertoncello, R. (Comp.) (2008). Turismo y geografía: lugares y patrimonio natural-cultural de la Argentina. Buenos Aires: CICCUS.

» Bertoncello, R. (2010). Turismo y patrimonio, entre la cultura y el negocio. En M. Duarte y M. Ramos (Orgs.), Geografía, turismo e patrimonio cultural (pp. 33-53). San Pablo: Annablume.

» Bertoncello, R. (2017). Prólogo. En A. Pinassi, Patrimonio cultural, turismo y recreación. El espacio vivido de los bahienses desde una perspectiva geográfica (pp. 10-16). Bahía Blanca: Ediuns.

» Bróndolo, M. y Zinger, A. (1978). Transportes, puertos y comunicaciones. En F. Weinberg (Dir.), Manual de historia de Bahía Blanca (pp. 207-222). Bahía Blanca: Universidad Nacional del Sur.

»Choay, F. (2007). Alegoría del patrimonio. Barcelona: Gustavo Gili.

»Consejo Internacional de Monumentos y Sitios (ICOMOS). (1999). Carta internacional sobre turismo cultural: la gestión del turismo en los sitios con patrimonio significativo. México: ICOMOS-UNESCO.

»Consorcio de Gestión del Puerto de Bahía Blanca. (2017). La Bahía... razones de una identidad. Recuperado de http://puertobahiablanca.com/identidad/index.php

»Crespí Vallbona, M. y Planells Costa, M. (2003). Patrimonio cultural. Madrid: Síntesis.

» Giop, M. y Flores, F. (2017). Discursos patrimoniales y narrativas espaciales. Historias de una localidad lujanense (Buenos Aires, Argentina) (1864-2016). En II Congreso Internacional de Geografía Urbana. Universidad Nacional de Luján, Lujan, Argentina.

" Gutiérrez, R. (2014). Repensando el patrimonio desde América Latina. En J. Zingoni y A. Pinassi (Comps.), Gestión del patrimonio urbano. Textos de cátedra (I) (pp. 63-80). Bahía Blanca: Ediuns.

" Gutiérrez, R. (2017). Repensando el patrimonio desde América Latina. En A. Novacovsky y F. París (Comps.), Textos de Cátedra V, Maestría en Gestión e Intervención del Patrimonio Arquitectónico y Urbano (pp. 9-25). Mar del Plata: Universidad Nacional de Mar del Plata.

» Harvey, D. (2005). El arte de la renta: la globalización y la mercantilización de la cultura. En D. Harvey y N. Smith. Capital financiero, propiedad inmobiliaria y cultura (pp. 29-57). Barcelona: Universidad Autónoma de Barcelona.

» Hernández Hernández, F. (2002). El patrimonio cultural: la memoria recuperada. Asturias: Trea.

» Manero Miguel, F. y García Cuesta, J. (Coords.) (2016). Patrimonio cultural y desarrollo territorial. Navarra: Thomson Reuters.

" Municipalidad de Bahía Blanca (1986). Reformulación del plan de desarrollo urbano de Bahía Blanca. Bahía Blanca: Municipalidad de Bahía Blanca. 
» Municipalidad de Bahía Blanca (1992). Inventario del patrimonio arquitectónico y urbano del Partido de Bahía Blanca. Bahía Blanca: Municipalidad de Bahía Blanca e Instituto Argentino de Investigaciones de Historia de la Arquitectura y del Urbanismo.

» Norrild, J. (2005). Aspectos generales del patrimonio. En R. Schlüter (Ed.), Turismo y patrimonio gastronómico. Una perspectiva (pp. 9-35). Buenos Aires: CIET.

» Organización de las Naciones Unidas para la Educación, la Ciencia y la Cultura (UNESCO). (1972). Convención sobre la protección del patrimonio mundial cultural y natural. París: UNESCO.

» Pardo Abad, C. (2008). Turismo y patrimonio industrial. Madrid: Síntesis.

»Pinassi, A. (2016). La configuración de un nuevo espacio turístico recreativo a través de la valorización del patrimonio cultural. El caso de Bahía Blanca (tesis de doctorado). Universidad Nacional del Sur, Departamento de Geografía y Turismo, Bahía Blanca, Argentina.

"Pinassi, A. (2017). Patrimonio cultural, turismo y recreación. El espacio vivido de los bahienses desde una perspectiva geográfica. Bahía Blanca: Ediuns.

» Prats, L. (1997). Antropología y patrimonio. Barcelona: Ariel.

»Prats, L. (1998). El concepto de patrimonio cultural. Política y Sociedad, 27, 63-76.

» Prats, L. (2005). Concepto y gestión del patrimonio local. Cuadernos de Antropología Social, 21, 17-35.

»Prats, L. (2006). La mercantilización del patrimonio: entre la economía turística y las representaciones identitarias. PH, Boletín del Instituto Andaluz del Patrimonio Histórico, $58,72-80$.

» Prats, L. (2012). El patrimonio en tiempos de crisis. Revista Andaluza de Antropología, 2, 68-85.

» Prats, L. (2014). El carácter magmàtic del patrimoni etnològic. Etnologia: Revista D’Etnologia de Catalunya, 39, 152-159.

»Puliafito, C. (2013). Ciudad-Puerto de Bahía Blanca: evolución y futuro del gigante portuario. Veinte años de autonomía, cinco siglos de historia. Buenos Aires: Servicios Directorio.

»Querol, M. (2010). Manual de gestión del patrimonio cultural. Madrid: Akal.

»Santana Talavera, A. (2002). Mirar y leer: autenticidad y patrimonio cultural para el consumo turístico. En VI Encuentro Nacional de Turismo con Base Local. Universidade Católica Dom Bosco (UCDB), Campo Grande, Brasil.

»Smith, L. (2011). El espejo patrimonial ¿ilusión narcisista o reflexiones múltiples? Antípoda, 12, 39-63.

»Troncoso, C. (2008). Creando un lugar turístico y patrimonial: las transformaciones de la Quebrada de Humahuaca a partir de los procesos de construcción de atractividad turística y patrimonialización (tesis de doctorado). Universidad de Buenos Aires, Facultad de Filosofía y Letras, Buenos Aires, Argentina.

"Troncoso, C. (2012). Turismo y patrimonio en la Quebrada de Humahuaca. Lugar, actores y conflictos en la definición de un destino turístico argentino. Tenerife: Pasos Edita.

» Troncoso, C. y Almirón, A. (2005). Turismo y patrimonio. Hacia una relectura de sus relaciones. Aportes y Transferencia, 9(1), 56-74.

»Viñuales, G. y Zingoni, J. (1990). Patrimonio urbano y arquitectónico de Bahía Blanca. Bahía Blanca: La Nueva Provincia. 


\section{Otras fuentes consultadas}

» Honorable Cámara de Diputados de la Nación (2002). Ley № 25580: declárase monumento histórico nacional a la usina General San Martín, ubicado en la ruta nacional 252-acceso a puertos-de la localidad de Ingeniero White, partido de Bahía Blanca. Recuperado de http://servicios.infoleg.gob.ar/infolegInternet/anexos/700oo-74999/74094/norma.htm

» Honorable Cámara de Diputados de la Nación (2014). Decreto № 2181: declaraciones de Monumentos Históricos Nacionales. Recuperado de http://servicios.infoleg.gob.ar/infolegInternet/anexos/235000-239999/238854/norma.htm

» Honorable Concejo Deliberante de Bahía Blanca (1994). Creación del Registro Municipal para la Preservación del Patrimonio Arquitectónico y Urbano del Partido de Bahía Blanca. Recuperado de http://www.bahiablanca.gov.ar/digesto/Ordenanza1.html?ord=7959

»La Nueva (2018a). Habrá un concurso para recuperar un sitio emblemático del puerto. Recuperado de https://www.lanueva.com/nota/2018-7-28-6-30-56-habra-un-concursopara-recuperar-un-sitio-emblematico-del-puerto

»La Nueva (2018b). Se viene la recuperación del histórico bulevar de acceso al puerto local. Recuperado de http://www.lanueva.com/nota/2018-6-24-6-30-38-recuperacion-delbulevar-guido-nuevo-paso-para-unir-a-bahia-con-su-puerto.

» Open Street Map (2017). Recuperado de https://www.openstreetmap.org/ 